\title{
TU/e EmonOWEN

\section{Atomic-scale structure and photoluminescence of InAs quantum dots in GaAs and AlAs}

\section{Citation for published version (APA):}

Offermans, P., Koenraad, P. M., Wolter, J. H., Pierz, K., Roy, M., \& Maksym, P. A. (2005). Atomic-scale structure and photoluminescence of InAs quantum dots in GaAs and AIAs. Physical Review B, 72(16), $165332-$ 1/6. [165332]. https://doi.org/10.1103/PhysRevB.72.165332

DOI:

10.1103/PhysRevB.72.165332

Document status and date:

Published: 01/01/2005

\section{Document Version:}

Publisher's PDF, also known as Version of Record (includes final page, issue and volume numbers)

\section{Please check the document version of this publication:}

- A submitted manuscript is the version of the article upon submission and before peer-review. There can be important differences between the submitted version and the official published version of record. People interested in the research are advised to contact the author for the final version of the publication, or visit the $\mathrm{DOI}$ to the publisher's website.

- The final author version and the galley proof are versions of the publication after peer review.

- The final published version features the final layout of the paper including the volume, issue and page numbers.

Link to publication

\section{General rights}

Copyright and moral rights for the publications made accessible in the public portal are retained by the authors and/or other copyright owners and it is a condition of accessing publications that users recognise and abide by the legal requirements associated with these rights.

- Users may download and print one copy of any publication from the public portal for the purpose of private study or research.

- You may not further distribute the material or use it for any profit-making activity or commercial gain

- You may freely distribute the URL identifying the publication in the public portal.

If the publication is distributed under the terms of Article 25fa of the Dutch Copyright Act, indicated by the "Taverne" license above, please follow below link for the End User Agreement:

www.tue.nl/taverne

Take down policy

If you believe that this document breaches copyright please contact us at:

openaccess@tue.nl

providing details and we will investigate your claim. 


\title{
Atomic-scale structure and photoluminescence of InAs quantum dots in GaAs and AlAs
}

\author{
P. Offermans,* P. M. Koenraad, and J. H. Wolter \\ Department of Semiconductor Physics, Eindhoven University of Technology, P. O. Box 513, NL-5600 MB Eindhoven, The Netherlands \\ K. Pierz \\ Physikalisch-Technische Bundesanstalt, Bundesallee 100, 38116 Braunschweig, Germany
}

M. Roy and P. A. Maksym

Department of Physics and Astronomy, University of Leicester, Leicester, LE1 7RH, United Kingdom

(Received 15 June 2005; revised manuscript received 22 August 2005; published 25 October 2005)

\begin{abstract}
We have determined the size, shape, and composition of InAs/GaAs quantum dots (QDs) and InAs QDs embedded in an AlAs barrier by cross-sectional scanning tunneling microscopy. The outward relaxation and lattice constant of the cleaved surface of the QDs and their wetting layers were calculated using continuum elasticity theory and compared with experimental data in order to determine the indium concentration of the dots. Based on the structural results we have calculated the electronic ground states of the dots using a single band, effective mass approach. We find that the calculated ground state photoluminescence energy of the InAs/GaAs dots is in excellent agreement with the measured energy. The observed large width of the PL spectrum of InAs/AlAs dots can be attributed to $\Gamma-\Gamma$ electron-hole recombination within an ensemble of dots with sizes varying between $2.4-4.2 \mathrm{~nm}$ in height and $10-20 \mathrm{~nm}$ along the base diagonal. We find that the electron-hole wave function overlap of small InAs/AlAs QDs is 7.6 times larger than that of InAs/GaAs QDs grown under the same conditions. This supports the explanation of the long decay times in InAs/AlAs dots by an enhanced exciton exchange splitting.
\end{abstract}

DOI: 10.1103/PhysRevB.72.165332

PACS number(s): 68.37.Ef, 68.65.Hb, 78.67.Hc, 81.07.Ta

\section{INTRODUCTION}

Self-assembled InAs quantum dots (QDs) embedded in AlAs barriers are of interest because of their large confinement potential ${ }^{1,2}$ compared to InAs/GaAs QDs and their consequent suitability for resonant tunneling devices. ${ }^{3,4}$ Despite the almost identical lattice mismatch, the formation of InAs QDs in AlAs differs considerably from that in GaAs. This is due to the larger Al-In bond strength, which reduces In migration on the AlAs surface, leading to a larger density and smaller size of InAs/AlAs QDs. ${ }^{5}$ It is these differences in size distribution, together with the larger confinement potential, that are thought to cause the large differences in the observed photoluminescence (PL) spectra between the two types of QD. ${ }^{3,5,6}$ However, the relationship between the differences in the spectra, and the properties of the two dot types is not quantitatively understood because of a lack of detailed structural information on buried InAs/AlAs QDs. ${ }^{7}$ Experimental and theoretical investigation of this topic is the subject of the present work.

We determine the shape, size, and composition of the QDs by cross-sectional scanning tunneling microscopy (X-STM). We show the composition variation in an InAs/AlAs dot and confirm the inverted trumpetlike indium distribution that has been assumed in InAs/GaAs QDs. ${ }^{8-11}$ Based on the X-STM results we then use a single band effective mass approach to calculate the electron and hole states and estimate the ground state PL energy. Our results compare favorably with the measured PL spectra and indicate that the large difference in the PL spectra of InAs/GaAs and InAs/AlAs QDs can be explained by the structural properties of the dots. Finally, in the light of these results, we discuss the mechanisms that have been suggested for the recently observed extremely long (microsecond) PL decay times of the InAs/AlAs dots. ${ }^{12-14}$

\section{EXPERIMENT}

The QDs were grown by molecular-beam epitaxy on doped GaAs (100) wafers. During growth of the QDs the substrate temperature was maintained at $500{ }^{\circ} \mathrm{C}$. Each $1.9 \pm 0.1$ monolayer (ML) of InAs was grown in a cycled way, i.e., with a $3 \mathrm{~s}$ pause after each deposition of $0.25 \mathrm{ML}$, at a low growth rate of $0.043 \mathrm{ML} / \mathrm{s}$. After a $500 \mathrm{~nm} n$-doped $\left(1 \times 10^{18} \mathrm{~cm}^{-2}\right) \mathrm{GaAs}$ buffer layer the following sequence was grown: $20 \mathrm{~nm} \mathrm{GaAs} / 1.9 \mathrm{ML}$ InAs/40 nm GaAs/1.9 ML InAs/40 nm GaAs/50 nm n-doped GaAs/20 nm GaAs/4 $\times(20 \mathrm{~nm} \mathrm{AlAs} / 1.9 \mathrm{ML}$ InAs/20 nm AlAs/40 nm GaAs).

To reduce interface roughness, the bottom AlAs barriers were grown at $600{ }^{\circ} \mathrm{C}$ followed by a growth interruption prior to InAs deposition.

The X-STM measurements have been performed in an ultrahigh-vacuum (UHV) chamber with base pressure less than $2 \times 10^{-11}$ Torr on the UHV-cleaved (110) crosssectional surface. The photoluminescence measurements were performed in an $\mathrm{He}$ cryostat at $7 \mathrm{~K}$ under $\mathrm{cw}$-excitation $(\lambda=514 \mathrm{~nm})$ from an $\mathrm{Ar}^{+}$laser.

\section{RESULTS AND DISCUSSION}

\section{A. Structure}

In Fig. 1 we show a large scale filled-states topography X-STM image of the cleaved structure. Three layers of QDs embedded in AlAs barriers and two layers of QDs grown on 


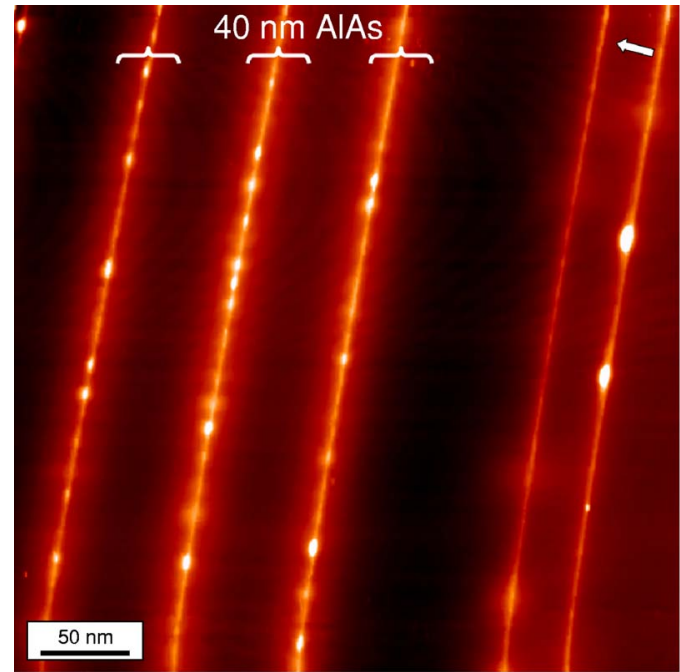

FIG. 1. (Color online) Filled states topography X-STM image of three QD layers embedded in 40-nm-thick AlAs barriers and two QD layers grown in GaAs, $V_{\text {sample }}=-3 \mathrm{~V}$. The arrow indicates the growth direction.

GaAs are visible in the image. Compared to the dots grown on GaAs, the QDs embedded in AlAs barriers show a smaller size and have a significantly larger density of about 3 $\times 10^{11} \mathrm{~cm}^{-2}$. This has been attributed to reduced diffusion of In adatoms on the AlAs surface due to a higher surface roughness and the larger Al-In bond strength. ${ }^{5}$

When the structure is cleaved, the cleavage surface deforms to reduce the built-in strain energy of the buried quantum dot. This surface relaxation is characterized by an outward displacement of the surface (outward relaxation) and a change in the lattice spacing in the plane of the surface. Regions under compressive strain bulge outward, while tensile strain depresses the surface. The outward displacement and change in the lattice spacing can be used to determine the indium composition of the strained quantum dots by comparing the experimental data with the calculated strain relaxation using elasticity theory.

By imaging at a high voltage $\left(V_{\text {sample }}=-3 \mathrm{~V}\right)$, electronic contributions to the contrast in the image are minimized and only the true outward relaxation due to the lattice mismatch (7\%) between the InAs and surrounding GaAs or AlAs is imaged. ${ }^{15}$ This is the reason why the AlAs barriers do not appear as dark layers.

In X-STM the QDs are cleaved at a random position with respect to the center of the QD. However, the position of the cleavage plane determines which part of the QD remains after cleavage and, therefore, the contribution to the outward relaxation of the surface. We have imaged more than 20 QDs and selected the largest ones for analysis. It can then be assumed that these QDs are cleaved near their middle, which enables a consistent modeling of their size, shape, and composition.

In Figs. 2(a) and 2(b) we compare high-voltage filledstates topography images of individual InAs/GaAs and InAs/AlAs QDs, respectively. The images show the outward relaxation of the cleaved QDs, which varies with the local indium distribution in the QDs. The outward relaxation of

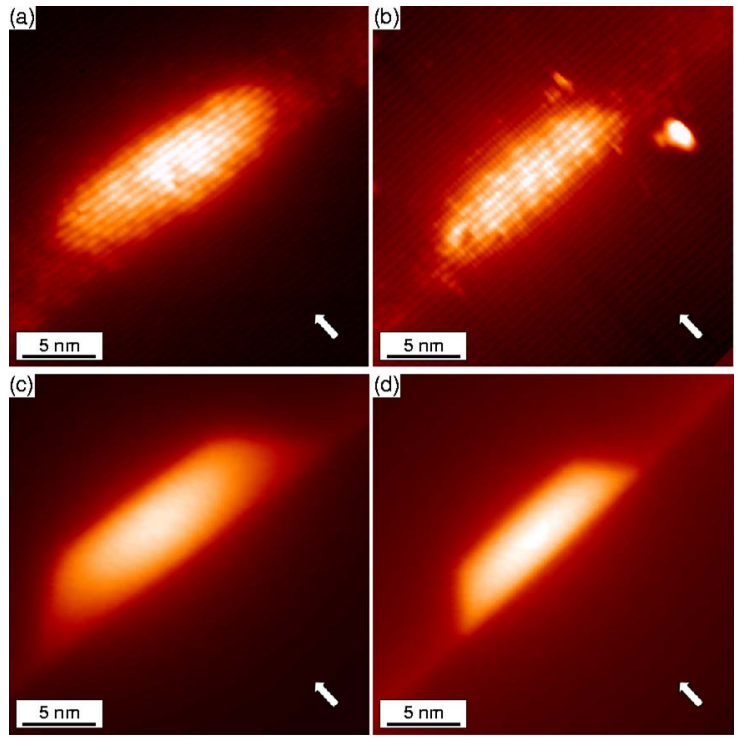

FIG. 2. (Color online) Filled states topography X-STM images of (a) InAs/GaAs QD, (b) InAs/AlAs QD. (c) and (d) show the calculated outward relaxation corresponding to (a) and (b). The height scale for (a) and (c) is 0 (dark) to $600 \mathrm{pm}$ (bright). The height scale for (b) and (d) is 0 (dark) to $450 \mathrm{pm}$ (bright). $V_{\text {sample }}=-3 \mathrm{~V}$. The arrows indicate the growth direction.

the InAs/GaAs dot indicates a lower indium concentration in the corners of the trapezium-shaped cross section compared to the center, while the indium distribution in the InAs/AlAs dot is more homogeneous.

We calculated the outward relaxation and the strain distribution of the cleaved QDs with the finite element package ABAQUS, which is based on continuum elasticity theory. The QD shape was taken to be a cleaved truncated pyramid which is consistent with the observed cross section and earlier work. ${ }^{16,17}$ The QD sizes were determined from the X-STM measurements while the indium distribution was varied in order to get the optimal fit to the measured outward relaxation. The best results were obtained by allowing the modeled QDs to be cleaved at a plane $1 \mathrm{~nm}$ above their diagonal. The diagonal base length and the height of the InAs/GaAs QD is $28.4 \mathrm{~nm}$ and $6 \mathrm{~nm}$, respectively. For the InAs/AlAs QD the diagonal base length is $19 \mathrm{~nm}$ and the height is $4.2 \mathrm{~nm}$. The calculated relaxation of the cleaved surface of the QDs is shown in Figs. 2(c) and 2(d) using the same height scale as in the corresponding X-STM images, Figs. 2(a) and 2(b).

Figure 3 shows the measured and calculated outward relaxation profiles [3(a) and 3(b)] and lattice constant profiles $[3(\mathrm{c})$ and $3(\mathrm{~d})]$ taken in the growth direction through the center of the QDs. The lattice constant profiles are derived from Figs. 2(a) and 2(b) by ensemble averaging of the spacing between the atomic rows of the cleaved (110) surface measured as a function of distance in the growth direction. The ensemble averaging was performed on lattice constant profiles taken within a $2 \mathrm{~nm}$ region around the central axis of the QDs. From the change in lattice constant, which is determined by the strain distribution in and around the QDs, it can be seen that there is compressive strain above and below the 
(a)

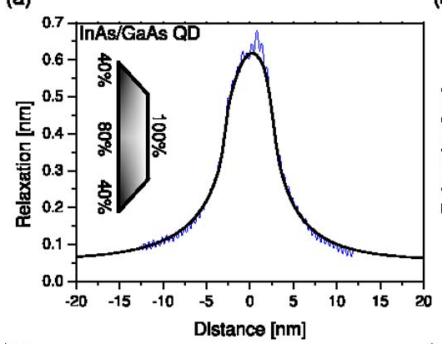

(c)

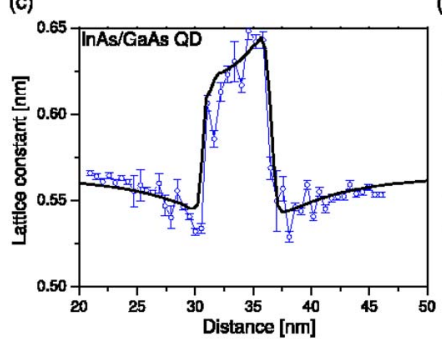

(e)

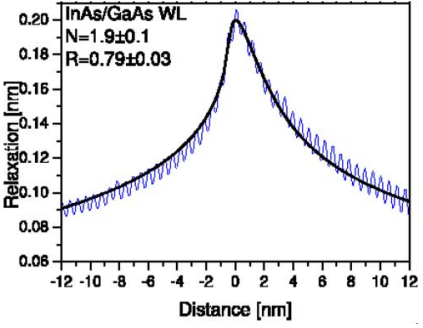

(f)

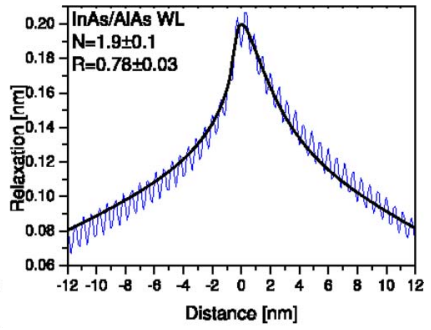

FIG. 3. (Color online) Calculated and measured outward relaxation profiles through the center of the QD in the growth direction for an InAs/GaAs QD (a) and an InAs/AlAs QD (b). (c) and (d) are the corresponding calculated and measured lattice constant profiles. (e) and (f) are measured and calculated outward relaxation profiles of the segregated wetting layers in GaAs (e) and AlAs (f). The growth direction is from left to right.

QDs. For the InAs/GaAs QD, there is a clear increase in lattice constant towards the top of the QD, which indicates an increasing indium concentration. This can also be seen by the slight asymmetry in the relaxation profile of the InAs/GaAs QD. From X-STM and photocurrent experiments, it has been shown that low growth-rate InAs/GaAs QDs have an increasing indium concentration in the growth direction. ${ }^{17,18}$ However, other groups have reported InGaAs QDs with laterally nonuniform indium compositions showing an inverted-triangle, trumpet, or truncated reversed-cone shape. ${ }^{8-11}$ We find that the indium distribution of our lowgrowth rate InAs/GaAs QDs mostly resembles the trumpet shape proposed in Ref. 9 which we describe with a linear gradient in both the growth direction and lateral direction, as shown by the inset in Fig. 3(a). Along the center of the QD, the indium concentration $x$ of the $\operatorname{In}_{x} \mathrm{Ga}_{1-x}$ As alloy changes from $x=0.8$ at the base to $x=1.0$ at the top of the QD. The gradient in the lateral direction depends on the position along the growth direction. At the base of the QD, $x$ varies linearly from 0.8 to 0.4 from the core to the perimeter, while at the top of the QD it remains constant. For the InAs/AlAs QD best fit results were obtained with an indium composition decreasing from $x=0.85$ at the base to $x=0.70$ at the top of the QD, as shown by the inset in Fig. 3(b). In this case there

is no evidence for a lateral gradient in the indium composition.

We attribute the observed differences in indium composition and size of the QDs in GaAs and AlAs to the combined effects of (a) the reduced diffusion of In on AlAs compared to GaAs, (b) the reduced intermixing for the InAs WL on AlAs, and (c) the capping process. It is known that the indium accumulation in QDs is determined by strain minimization during growth. ${ }^{19,20}$ However, the preferential indium aggregation at the In-rich region of the dot is limited by the lateral diffusion of indium in the case of growth on the AlAs substrate, which results in a reduced QD size and increased QD density. ${ }^{5}$

Furthermore, it has been shown ${ }^{21}$ that there is less intermixing for an InAs WL on AlAs compared to an InAs WL on GaAs. Together with the reduced mobility of In on AlAs, this explains the homogeneous indium distribution in the base of the InAs/AlAs QDs. It has been proposed that the growth of dots on AlAs is initiated by $2 \mathrm{D}$ islands which develop into small 3D islands when more InAs is deposited. ${ }^{21}$ This is in contrast to the growth of dots on GaAs where dot formation is initiated by small indium-rich nucleation centers which develop into trumpet-shaped indium distributions by the preferential diffusion of In to the apex of the dot. ${ }^{9}$ These growth mechanisms are supported by our observation of the indium distribution inside the dots.

The observed decrease of the indium concentration toward the top of the InAs/AlAs QD might be caused by the residual incorporation of AlAs in the top of the dot during the capping process. In order to reduce the total strain field in the QD some capping material is incorporated in the top of the dot. In the case of GaAs capping, the diluted part of the dot is very mobile and therefore disappears very quickly during capping, causing leveling of the QDs. ${ }^{22}$ The InAs diluted by $\mathrm{Al}$, however, is much less mobile due to the stronger Al-In bond strength and therefore more diluted material at the top remains in place.

Finally, the difference in the formation of QDs in AlAs and GaAs cannot be attributed to a different vertical indium segregation process in AlAs and GaAs. An image of the vertical segregation of the wetting layer of the InAs/AlAs dots structure is shown in Fig. 4. Figure 3 shows the measured and calculated relaxation profiles for the segregated wetting layer in AlAs (e) and GaAs (f). The calculated relaxation profiles have been derived using the phenomenological model of Muraki et al. ${ }^{23}$ to describe the indium segregation profile. We find a segregation coefficient of $R=0.79 \pm 0.03$ and $R=0.78 \pm 0.03$ for GaAs and AlAs, respectively, in agreement with Ref. 24. This indicates that strain rather than the chemical bond strength determines the vertical indium segregation.

\section{B. Photoluminescence}

In the following we will show that we can calculate the ground state photoluminescence energy from the measured size, shape, and composition of the QDs. Based on our structural analysis we have calculated the single particle bound electron and hole states in both the InAs/GaAs and 


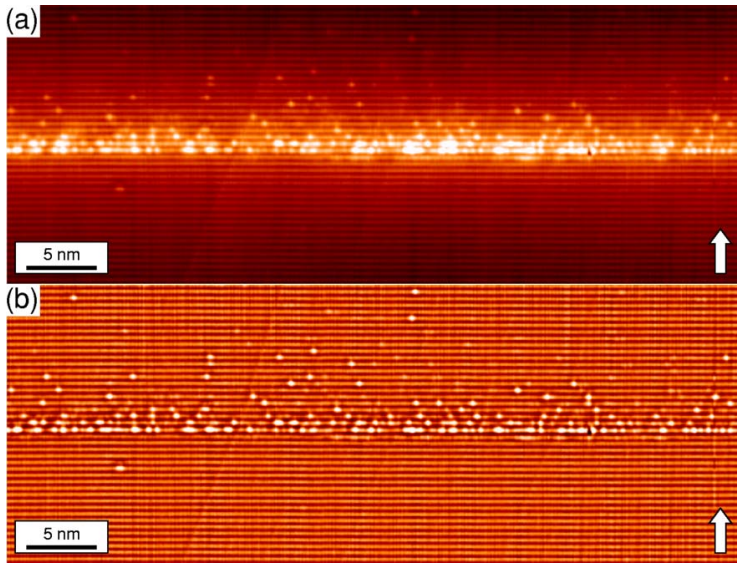

FIG. 4. (Color online) Filled states topography X-STM image of the InAs/AlAs segregated wetting layer (a). By using an inverse fourier filter, the background contrast is removed (b). The arrow indicates the growth direction.

InAs/AlAs QDs shown in Fig. 2. We use the electron and hole ground state energies to estimate the energy of the ground state exciton and compare this to the photoluminescence results shown in Fig. 5. The InAs/GaAs dots show a second PL peak around $1.13 \mathrm{eV}$, which we attribute to a bimodal size distribution, since it does not disappear at low excitation density, nor increase considerably at high excitation density compared to the first peak. The bimodal size distribution was confirmed by atomic force microscopy measurements on the top (uncapped) layer. The broad PL spectrum of InAs/AlAs dots has been attributed to a unimodal dot size distribution. ${ }^{14}$

The calculation of the bound electron and hole states is performed with the method of Roy and Maksym ${ }^{25}$ in which the single band, effective mass Hamiltonian is diagonalized in a basis of harmonic oscillator functions. Within this approximation the strain dependence of the band gap is included exactly in the Hamiltonian, while the strain dependence of the electron and hole mass is included with perturbation theory. ${ }^{26,27}$ In our calculation the dot structures and composition variations are taken directly from our X-STM measurements. We linearly interpolate the band parameters and effective masses to obtain the values relevant to the InGaAs and InAlAs alloy material in the QDs.

It is well known that a single band calculation adequately describes the electron states and the hole ground state ${ }^{27}$ in InAs/GaAs QDs. For the calculation of the electron and hole ground state of the InAs/GaAs QD shown in Fig. 2(a), we used the band parameters given in Ref. 27, and converged the calculated electron and hole energies to within $0.1 \mathrm{meV}$. We estimate the ground state photoluminescence energy from the electron $E_{e}$ and hole $E_{h h}$ ground state energies as $\hbar \omega \approx\left|E_{e}\right|-\left|E_{h h}\right|-10 \mathrm{meV}$, where the $10 \mathrm{meV}$ is a rough approximation to the electron-hole interaction energy. With $E_{e}=1275.9 \mathrm{meV}$ and $E_{h h}=222.3 \mathrm{meV}$, we find a PL energy of $\hbar \omega \approx 1044 \mathrm{meV}$, which corresponds to the observed PL peak position to within $15 \mathrm{meV}$ [shown by the arrow in Fig. $5(\mathrm{a})]$. Since we typically look for the largest dots in the $\mathrm{X}$-STM measurements, it is expected that the calculated PL energy is at the low energy side of the observed PL peak.
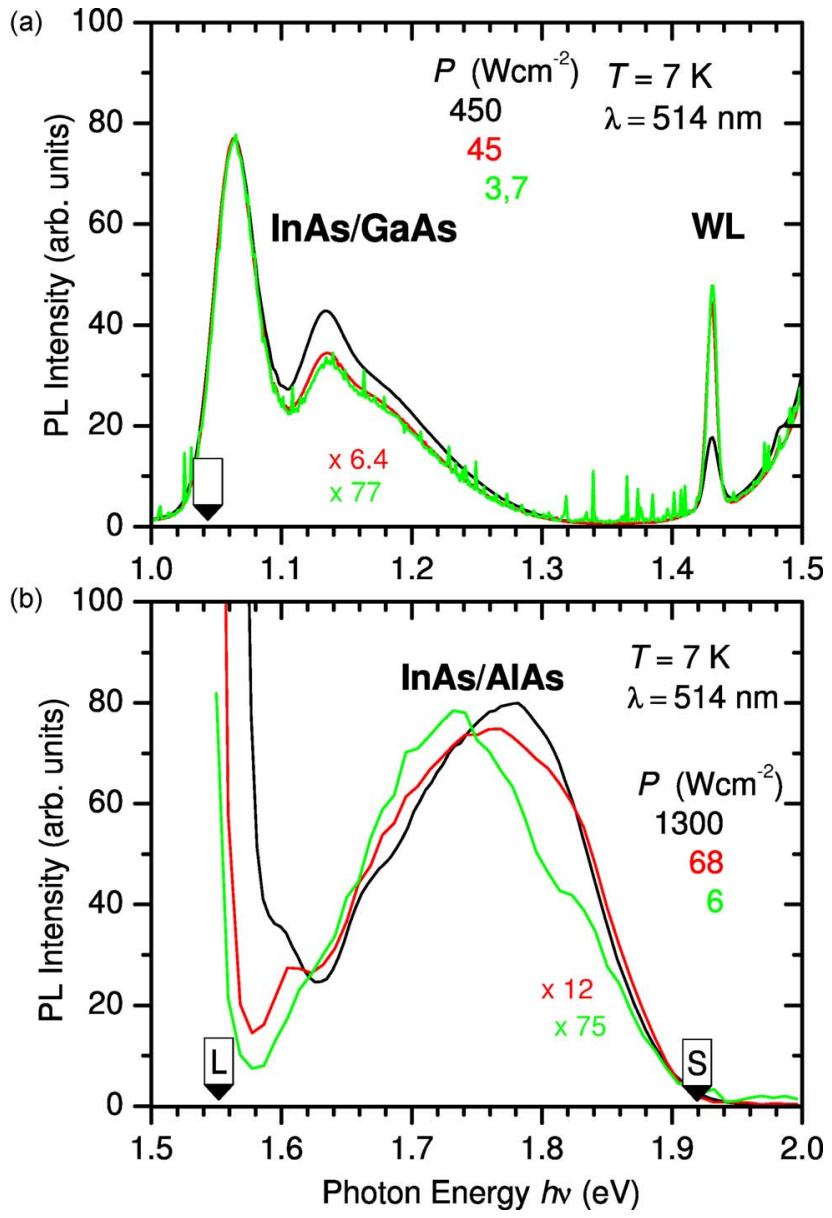

FIG. 5. (Color online) Photoluminescence (PL) of InAs/GaAs (a) and InAs/AlAs (b) dots measured at $7 \mathrm{~K}$. The double peak from the InAs/GaAs dots (a) indicates a bimodal size distribution which was confirmed by atomic force microscopy measurements of the uncapped dot layer at the sample surface. The calculated ground state PL energy is indicated by the arrow. The broad PL peak of the AlAs dots (b) can be attributed to an ensemble of dots with varying sizes. The large increase in PL intensity at the low energy end of the spectrum is due to bandgap recombination of the GaAs substrate. The arrows labeled L and S indicate the calculated ground state PL energy for large and small dots as discussed in the text.

In contrast to InAs/GaAs QDs, the electron states in InAs/AlAs QDs may be bound at the $\Gamma, X$, or $L$ point. We have calculated the electron and heavy hole single band envelope functions using the band parameters shown in Table I. The calculated electron and hole energies are converged to within $0.5 \mathrm{meV}$. The slower convergence in this type of QD, compared to the InAs/GaAs QDs, is due to the much larger confinement potential.

From the $\Gamma$ bound electron $\left(E_{e}=2175 \mathrm{meV}\right)$ and hole states $\left(E_{h}=611 \mathrm{meV}\right)$ in the InAs/AlAs QD shown in Fig. 2(b), we estimate a ground state PL energy of $\hbar \omega$ $\approx 1554 \mathrm{meV}$, which corresponds to the low energy side of the broad PL peak spectrum as shown by the "L" arrow in Fig. 5(b). As stated previously, this is to be expected since the analyzed InAs/AlAs dot is one of the largest in the sample, as observed by X-STM. 
TABLE I. Electron and hole parameters for AlAs and InAs. The deformation potentials $a_{c}$, at $X$ and $L$ are obtained from data given in Ref. 33. Electron parallel $\left(m_{e}^{\|}\right)$and perpendicular $\left(m_{e}^{z}\right)$ masses at $X$ and $L$ are taken from Ref. 28.

\begin{tabular}{|c|c|c|c|c|c|c|}
\hline \multirow{2}{*}{$\begin{array}{c}\text { Band } \\
\text { parameter }\end{array}$} & \multicolumn{3}{|c|}{ AlAs } & \multicolumn{3}{|c|}{ InAs } \\
\hline & $\Gamma$ & $X$ & $L$ & $\Gamma$ & $X$ & $L$ \\
\hline$E_{c}(\mathrm{eV})^{\mathrm{a}}$ & 3.13 & $2.42^{\mathrm{e}}$ & 2.46 & 1.27 & 2.29 & 1.99 \\
\hline$m_{e}^{\|}$ & $0.124^{\mathrm{b}}$ & 0.22 & 0.26 & $0.023^{\mathrm{f}}$ & 0.16 & 0.09 \\
\hline$m_{e}^{z}$ & $0.124^{\mathrm{b}}$ & 0.97 & 0.21 & $0.023^{\mathrm{f}}$ & 1.13 & 0.07 \\
\hline$a_{c}(\mathrm{eV})$ & $-5.64^{\mathrm{c}}$ & 4.30 & -1.31 & $-5.08^{f}$ & 1.50 & -2.31 \\
\hline$m_{h}^{\|}$ & $0.21^{\mathrm{d}}$ & & & $0.036^{\mathrm{f}}$ & & \\
\hline$m_{h}^{z}$ & $0.403^{\mathrm{d}}$ & & & $0.34^{\mathrm{f}}$ & & \\
\hline$a_{v}(\mathrm{eV})$ & $2.47^{\mathrm{c}}$ & & & $1.00^{\mathrm{f}}$ & & \\
\hline$b(\mathrm{eV})$ & $-1.5^{\mathrm{c}}$ & & & $-1.8^{\mathrm{f}}$ & & \\
\hline
\end{tabular}

a Band gaps taken from Ref. 28 and aligned according to Ref. 29. The values are relative to the AlAs valence band edge.

${ }^{b}$ From Ref. 30.

${ }^{c}$ From Ref. 29.

${ }^{\mathrm{d} F r o m ~ R e f . ~} 31$.

${ }^{\mathrm{e}}$ From Ref. 32.

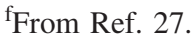

The broad PL spectrum of InAs/AlAs dots has been attributed to a spread in dot size. ${ }^{14}$ To investigate this, we repeated the calculation for smaller InAs/AlAs QDs with the same shape as the large dot and the same vertical variation in indium fraction from $85 \%$ to $70 \%$. For a dot with a height of $2.4 \mathrm{~nm}$ and diameter of $10.8 \mathrm{~nm}$, we find that the lowest $\Gamma$ bound state shifts up in energy by $246 \mathrm{meV}$ while the heavy hole ground state moves down by $120 \mathrm{meV}$. This results in a PL energy of $\hbar \omega \approx 1920 \mathrm{meV}$, which corresponds to the high energy side of the PL spectrum in Fig. 5(b), as indicated by the "S" arrow. It is difficult to determine the smallest dot size from cross-sectional images since the position of the cleavage plane is unkown. The existence of InAs/AlAs QDs as small as $8 \mathrm{~nm}$ in diameter has been reported in the literature,,${ }^{14}$ however, if we decrease the QD diameter below $10.8 \mathrm{~nm}$ we find that the lowest energy state at $\Gamma$ moves above the AlAs $X$ band edge, and is no longer confined to the dot. In such small QDs, the recombination becomes both spatially indirect and indirect in $k$ space, and is therefore expected to give only a weak contribution to the PL.

For the large QD, we find that the $L$ bound ground state is well above $(114 \mathrm{meV})$ the $\Gamma$ bound ground state. There is a large spread in the reported values for the position of the AlAs conduction band edge at $X{ }^{29,32}$ We find that the AlAs $X$ band edge may be either above or below the unstrained InAs $X$ band edge. In either case, the band edge deformation potential is positive at the $X$ point, ${ }^{33}$ and the large compressive strain in the QD lowers the dot $X$ band edge below that of the AlAs barrier, leading to a weakly bound state in the $X$ valley. Using the parameters shown in Table $\mathrm{I}$, we find that the $X$ bound electron state in the large InAs/AlAs QD, shown in Fig. 2(b), is $74 \mathrm{meV}$ above the $\Gamma$ bound electron state. In the small InAs/AlAs QD discussed earlier, the lowest $\Gamma$ bound electron state coincides with the AlAs $X$ band edge and is $123 \mathrm{meV}$ above the $X$ bound state. If we assume that the normally forbidden $\Gamma-X$ transitions $d o$ contribute to the PL, these transitions would only contribute in the narrow energy window between 1628 and $1796 \mathrm{meV}$. We therefore conclude that the large width of the PL peak shown in Fig. 5(b) is due to $\Gamma-\Gamma$ electron-hole recombination, with the low energy side of the PL peak determined by the largest dots, such as the one analyzed by X-STM, and the high energy side determined by the smallest dots that contribute to the PL. These could be either the smallest dots in the distribution or the smallest dots that have a direct $\Gamma-\Gamma$ transition.

It has been suggested that QDs without a bound electron state do not contribute to the PL because electrons can rapidly scatter via the $X$ states in the AlAs barrier to the surrounding GaAs matrix or to larger QDs, ${ }^{13}$ where $\Gamma-\Gamma$ transitions are allowed, as shown in Fig. 6. Repeated scattering would cause the extremely long decay times (microseconds)

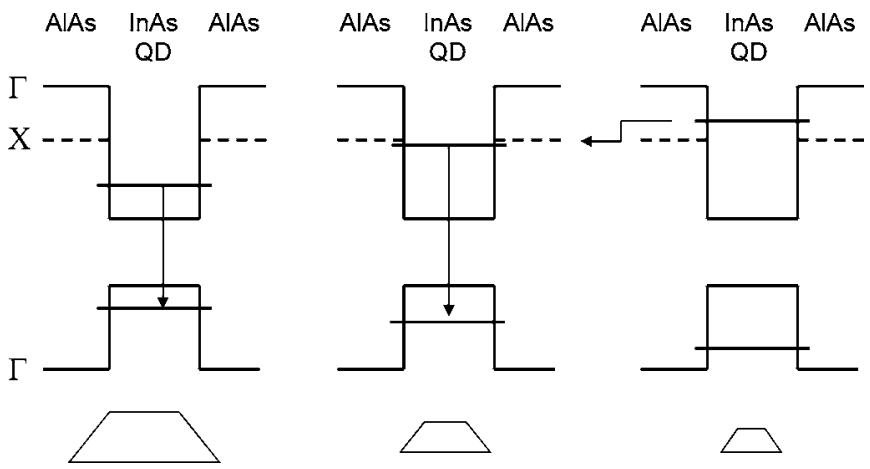

FIG. 6. Band scheme for differently sized InAs QDs embedded in an AlAs barrier, showing the shift of the lowest $\Gamma$ bound electron state above the AlAs $X$ conduction band edge with decreasing dot size. For the smallest dot the $\Gamma$ electron is no longer confined to the dot and may scatter to the surrounding GaAs matrix or to larger QDs (Ref. 13). 
observed in InAs/AlAs QDs. ${ }^{13}$ However, such long decay times have also been observed for excitation $(\hbar \omega=1.82 \mathrm{eV})$ below the AlAs $X$ conduction band edge. ${ }^{14}$ This has been explained by enhanced exchange splitting of strongly confined exciton levels in small InAs/AlAs QDs, ${ }^{14}$ which would lead to a dark ground state ${ }^{34}$ in the smallest InAs/AlAs QDs. Due to the high QD density, carriers could transfer from the small dots to larger ones ${ }^{6}$ with a smaller splitting, and eventually recombine. We have estimated the exchange splitting of the small InAs/AlAs QD discussed previously by calculation of the electron-hole wave function overlap $P$ $\sim \int \psi_{e}(\mathbf{r}) \psi_{h}(\mathbf{r}) d \mathbf{r} .{ }^{35}$ We find that this is 7.6 times larger than that of the InAs/GaAs QD, which will clearly lead to an enhancement in the exciton exchange splitting in InAs/AlAs QDs.

\section{CONCLUSION}

To summarize, we have determined the size, shape, and composition of InAs/GaAs QDs and InAs QDs embedded in an AlAs barrier, by X-STM. The outward relaxation and lattice constant of the cleaved surface of the QDs and their wetting layers were calculated using continuum elasticity theory and compared with experimental data in order to determine the indium concentration of the dots. Based on the structural results we have calculated the electronic ground states of the dots using a single band, effective mass approach. We find that the calculated ground state photoluminescence energy of the InAs/GaAs dots is in excellent agreement with the measured energy. The observed large width of the PL spectrum of InAs/AlAs dots can be attributed to $\Gamma-\Gamma$ electron-hole recombination within an ensemble of dots with sizes varying between $2.4-4.2 \mathrm{~nm}$ in height and $10-20 \mathrm{~nm}$ along the base diagonal. We find that the electronhole wave function overlap of small InAs/AlAs QDs is 7.6 times larger than that of InAs/GaAs QDs grown under the same conditions. This supports the explanation of the long decay times in InAs/AlAs dots by an enhanced exciton exchange splitting.
*Electronic address: p.offermans@tue.nl

${ }^{1}$ J. L. Movilla, J. I. Climente, and J. Planelles, J. Appl. Phys. 94, 4515 (2003).

${ }^{2}$ J. M. García, T. Mankad, P. O. Holtz, P. J. Wellman, and P. M. Petroff, Appl. Phys. Lett. 72, 3172 (1998).

${ }^{3}$ I. Hapke-Wurst, U. Zeitler, H. W. Schumacher, R. J. Haug, K. Pierz, and F. J. Ahlers, Semicond. Sci. Technol. 14, L41 (1999).

${ }^{4}$ A. S. G. Thornton, T. Ihn, P. C. Main, L. Eaves, and M. Henini, Appl. Phys. Lett. 73, 354 (1998).

${ }^{5}$ K. Pierz, Z. Ma, U. F. Keyser, and R. J. Haug, J. Cryst. Growth 249, 477 (2003).

${ }^{6}$ U. H. Lee, D. Lee, H. G. Lee, S. K. Noh, J. Y. Leem, and H. J. Lee, Appl. Phys. Lett. 74, 1597 (1999).

${ }^{7}$ F. Ferdos, S. Wang, Y. Wei, H. Sadeghi, Q. Zhao, and A. Larsson, J. Cryst. Growth 251, 145 (2003).

${ }^{8}$ N. Liu, J. Tersoff, O. Baklenov, A. L. Holmes Jr., and C. K. Shih, Phys. Rev. Lett. 84, 334 (2000).

${ }^{9}$ M. A. Migliorato, A. G. Cullis, M. Fearn, and J. H. Jefferson, Phys. Rev. B 65, 115316 (2002).

${ }^{10}$ O. Wolst, M. Kahl, M. Schardt, S. Malzer, and G. H. Dohler, Physica E (Amsterdam) 17, 554 (2003).

${ }^{11}$ A. Lenz, R. Timm, H. Eisele, C. Hennig, S. K. Becker, R. L. Sellin, U. W. Pohl, D. Bimberg, and M. Dhne, Appl. Phys. Lett. 81, 5150 (2002).

${ }^{12}$ D. Sarkar, H. P. van der Meulen, J. M. Calleja, J. M. Becker, R. J. Haug, and K. Pierz, Phys. Rev. B 71, 081302(R) (2005).

${ }^{13}$ P. Dawson, Z. Ma, K. Pierz, and E. O. Gbel, Appl. Phys. Lett. 81, 2349 (2002).

${ }^{14}$ T. S. Shamirzaev, A. M. Gilinksnky, A. K. Bakarov, A. I. Toporov, D. A. Tenne, K. S. Zhuravlev, C. von Borczyskowski, and D. R. T. Zahn, JETP Lett. 77, 389 (2003).

${ }^{15}$ R. M. Feenstra, Physica B 273, 796 (1999).

${ }^{16}$ O. Flebbe, H. Eisele, T. Kalka, F. Heinrichsdorff, A. krost, D. Bimberg, and M. Dähne-Prietsch, J. Vac. Sci. Technol. B 17, 169 (1999).
${ }^{17}$ D. M. Bruls, J. W. A. M. Vugs, P. M. Koenraad, H. W. M. Salemink, J. H. Wolter, M. Hopkinson, M. S. Skolnick, F. Long, and S. P. A. Gill, Appl. Phys. Lett. 81, 1708 (2002).

${ }^{18}$ P. W. Fry et al., Phys. Rev. Lett. 84, 733 (2000).

${ }^{19}$ T. Walther, A. G. Cullis, D. J. Norris, and M. Hopkinson, Phys. Rev. Lett. 86, 2381 (2001).

${ }^{20}$ E. Pehlke, N. Moll, A. Kley, and M. Scheffler, Appl. Phys. A 65, 525 (1997).

${ }^{21}$ P. Ballet, J. B. Smathers, and G. J. Salamo, Appl. Phys. Lett. 75, 337 (1999).

${ }^{22}$ Q. Gong, P. Offermans, R. Nötzel, P. M. Koenraad, and J. H. Wolter, Appl. Phys. Lett. 85, 5697 (2004).

${ }^{23}$ K. Muraki, S. Fukatsu, Y. Shiraki, and R. Ito, Appl. Phys. Lett. 61, 557 (1992).

${ }^{24}$ M. Schowalter, A. Rosenauer, D. Gerthsen, M. Arzberger, M. Bichler, and G. Abstreiter, Appl. Phys. Lett. 79, 4426 (2001).

${ }^{25}$ M. Roy and P. A. Maksym, Phys. Rev. B 68, 235308 (2003).

${ }^{26}$ L. R. C. Fonseca, J. L. Jimenez, J. P. Leburton, and R. M. Martin, Phys. Rev. B 57, 4017 (1998).

${ }^{27}$ J. A. Barker and E. P. O'Reilly, Phys. Rev. B 61, 13840 (2000).

${ }^{28}$ I. Vurgaftman, J. R. Meyer, and L. R. Ram-Mohan, J. Appl. Phys. 89, 5815 (2001)

${ }^{29}$ C. G. Van de Walle, Phys. Rev. B 39, 1871 (1989).

${ }^{30}$ W. P. Dumke, M. R. Lorenz, and G. D. Petit., Phys. Rev. B 5, 2978 (1972).

${ }^{31}$ P. Lawaetz, Phys. Rev. B 4, 3460 (1971).

${ }^{32}$ D. I. Westwood, M. L. Ke, F. Lelarge, F. Laruelle, and B. Etienne, Surf. Sci. 352-354, 802 (1996).

${ }^{33}$ S.-H. Wei and A. Zunger., Phys. Rev. B 60, 5404 (1999).

${ }^{34}$ J. M. Smith, P. A. Dalgarno, R. J. Warburton, A. O. Govorov, K. Karrai, B. D. Gerardot, and P. M. Petroff, Phys. Rev. Lett. 94, 197402 (2005).

${ }^{35}$ M. Bayer, A. Kuther, A. Forchel, A. Gorbunov, V. B. Timofeev, F. Schafer, J. P. Reithmaier, T. L. Reinecke, and S. N. Walck, Phys. Rev. Lett. 82, 1748 (1999). 